Portland State University

PDXScholar

Dissertations and Theses

Dissertations and Theses

Spring 1-1-2012

\title{
Stress Reactions of Division-I Track Athletes
}

Laura Elizabeth Skarda

Portland State University

Follow this and additional works at: https://pdxscholar.library.pdx.edu/open_access_etds

Part of the Orthopedics Commons, and the Sports Sciences Commons Let us know how access to this document benefits you.

Recommended Citation

Skarda, Laura Elizabeth, "Stress Reactions of Division-I Track Athletes" (2012). Dissertations and Theses. Paper 749.

https://doi.org/10.15760/etd.749

This Thesis is brought to you for free and open access. It has been accepted for inclusion in Dissertations and Theses by an authorized administrator of PDXScholar. Please contact us if we can make this document more accessible: pdxscholar@pdx.edu. 


\section{Stress Reactions of Division-I Track Athletes}

by

\section{Laura Elizabeth Skarda}

A thesis submitted in partial fulfillment of the requirements for the degree of

\section{Master of Science}

in

Health Studies

Thesis Committee:

Gary Brodowicz, Chair

James Wallis

Jonathan Huwe

Portland State University

(C)2012 


\begin{abstract}
Problem: Track and field athletes, along with cross-country athletes have multiple and back to back seasons, creating overuse injuries. Stress fractures or stress reactions to the bone are the overuse injuries focused on in this study and literature review. There is a lack of information in the literature regarding stress reactions.

Purpose: The goal of the study is to understand more information about stress reactions to bone and possibly increase the knowledge of health care professionals.

Methods: Three case studies were examined through pre-existing medical chart notes and athletic trainer's notes regarding the stress reactions. A literature review was also performed to provide further information about stress fractures and stress reactions.

Conclusions: There are multiple risk factors for stress injuries. All three of the athletes in the case studies are female, which is found to be a risk factor. Many risk factors need more studies to provide support. Magnetic Resonance Imaging (MRI) was obtained in all three case studies where there was found to be a stress reaction. These three females also had a recent increase in activity level and had similar symptoms to each other and what is found in the literature.
\end{abstract}




\section{Dedication}

My thesis is dedicated to my athletes, family and friends who have been supportive, but especially to my grandmother who passed away during my time at Portland State. She was the strongest and most amazing woman I have known. 


\section{Acknowledgements}

Without my three track and field athletes allowing me to analyze and share their anonymous stories my thesis would not be possible. I appreciate them immensely. They know who they are.

Gary, Jim and Jonathan, thank you so much for all of your patience and help throughout this whole process. Amazing thesis committee.

My parents have supported me in everything I have done. They believe in me even when I am unable to believe in myself.

My boyfriend was able to handle all of the ups and downs that came with my thesis, thank goodness. Love you boo. I finished it!

Big thank you to John Lawrence who helped me with my page numbering formatting. Life saver. 


\section{Table of Contents}
Abstract
Page i
Dedication
Page ii
Acknowledgements
Page iii
Chapter I Introduction
Page 1
Chapter II Methodology
Page 6
Chapter III Case Study \# 1
Page 7
Case Study \#2
Page 14
Case Study \#3
Page 19
Chapter IV Literature Review
Page 23
References
Page 42 


\section{Case Studies: Stress Reactions of Division I Track Athletes}

\section{CHAPTER I}

\section{Introduction}

\section{Statement of the Problem}

Track athletes have two competitive seasons back to back: an indoor season followed immediately by an outdoor season. Cross-country athletes participate in the distance portion of track, resulting in three competitive seasons in one year for these athletes. These intense periods of training and competition over this length of time increase the probability of overuse injuries. These injuries are either muscular or skeletal; however they can be both. For the purposes of this study, the focus will be on skeletal overuse injuries, commonly referred to as bone stress injuries or stress injuries. Stress injuries can refer to either a stress reaction or a stress fracture.

Use of the term stress fracture refers to a fracture line or break in continuity of the bone. Clinical findings include: a history of increased training or alteration in training plan, prior stress injury, pain or point tenderness over the fracture site, constant pain, $\mathrm{x}$ rays demonstrating a fracture line, or a positive bone scan. Stress reaction means there is no fracture line or break in continuity of the bone; however, the clinical symptoms are usually identical. X-ray images are not able to reveal an initial stress reaction. Therefore magnetic resonance imaging (MRI) or computerized tomography (CT) scan must be performed. However, a bone scan would still be positive in the case of a stress reaction. 
The etiology of stress injuries and their risk factors will be discussed in the literature review.

\section{Purpose of the Study}

The purpose of the study is to examine three cases of stress reactions in collegiate athletes, and describe how the symptoms presented, were treated, and how the stress reaction was managed in the athlete's return to activity. The research addresses how stress reactions are presented, diagnosed, treated, immobilized, rehabilitated and managed in returning to a pre-injury level of activity. The goal was to gain understanding about stress reactions to bone, which could presumably increase the knowledge of health care professionals who may find it necessary to identify and treat athletes with such an injury.

\section{Significance}

This study is significant because there is a distinct lack of information in the current body of literature focusing on stress reactions. Several studies and reviews have focused on stress fractures in military recruits (Shaffer \& Uhl, 2006; Rome, Handoll, \& Ashford, 2009; Giladi, Milgrom, Simkin, \& Danon, 1991) or athletes (Kelsey et al., 2007; Zeni, Street, Dempsey, \& Staton, 2000; Khan, Brukner, Kearney, Fuller, Bradshaw, \& Kiss, 1994; Bennell, Malcolm, Wark, \& Brukner, 1996; Sterling, Edelstein, Calvo, \& Webb II, 1992; Bennell, et al., 1998) but there is a dearth of information specifically about stress reactions (Burne, Mahoney, Forster, Koehle, Taunton, \& Khan, 2005; Shaffer \& Uhl, 2006). The description, exploration and analysis of the present case 
studies will presumably add to the body of knowledge for health care professionals with information useful in diagnosis, treatment and management of stress reactions.

\section{Limitations}

External validity is the primary limitation in this study. Female track and field athletes are the focus of these descriptive, explanative and analytic case studies. Therefore, the study may not be applicable to males, military recruits, or other athletes or laypersons outside of track and field.

\section{Definition of Key Terms}

Stress Fracture: A fracture line or break in the continuity of the bone as shown in x-ray, CT or MRI images, also possibly with a positive bone scan. Clinical exam findings including history involve pain and tenderness over the bone, possibly palpable bump in fracture area, a change in training--either increase in frequency, intensity or duration, a history of stress injury, and the pain is constant (Burne, Mahoney, Forster, Koehle, Taunton, \& Khan, 2005; Fredericson, Bergman, Hoffman, \& Dillingham, 1995).

Stress Reaction: There is no fracture line or break in the continuity of bone found in $\mathrm{x}-$ ray, CT or MRI images, however, a positive bone scan is found. Clinically the history and examination is similar to that of stress fracture (Fredericson, Bergman, Hoffman, \& Dillingham, 1995; Burne, Mahoney, Forster, Koehle, Taunton, \& Khan, 2005).

Stress Injury: Used to refer to either a stress reaction or a stress fracture. 
Magnetic Resonance Imaging (MRI): MRI uses a magnetic field and radio wave energy pulses to create internal pictures (Magnetic Resonance Imaging (MRI), 2009). This is helpful for diagnosis of musculoskeletal injuries, specifically for bone stress injuries because it is sensitive and specific about changes to bone and the soft tissues surrounding it (Burne, Mahoney, Forster, Koehle, Taunton, \& Khan, 2005; Lassus, Tulikoura, Salo, \& Santavirta, 2002). MRI is valuable for grading of bone stress injuries, and is thought to be most accurate in the first three weeks (Lassus, Tulikoura, Salo, \& Santavirta, 2002). There are two different images taken in an MRI, T1 and T2. T1 images have good spatial resolution, fat will appear bright and the interpreter is able to define anatomy (Bradley). T2 images cause water to appear bright and fat dark, and also known as the "pathology weighted" sequence (Bradley). When an MRI is read, what is dark on T1 and bright on T2 shows pathology, due to water and edema (Bradley).

Radiograph or X-ray: a form of electromagnetic radiation sending individual x-ray particles through the body to record images created during the x-ray (X-ray, 2012). Xray is best used to identify a true stress fracture or rule out a stress fracture in the differential diagnosis of stress injuries.

Computerized Tomography (CT): Combines a series of views of x-rays taken from multiple angles and utilizes computer processing to create images of the body that are cross-sectional (Staff, 2012).

Bone Scan: Nuclear imaging test to help diagnose and track several types of bone diseases (Staff, Bone Scan, 2012). There is an injection into the vein of the person receiving the bone scan of radioactive materials, and then when the scan is performed 
there are "hot spots" or areas of increased uptake of the radioactive material, meaning this area in the body is actively repairing (Staff, Bone Scan, 2012).

Differential diagnosis: A group of possible diagnoses prior to the actual diagnosis.

Diagnostic Imaging: Refers to any images such as X-ray, MRI, CT-scan or bone scans taken throughout the process of the clinical examination. 


\section{CHAPTER II}

\section{Methodology}

\section{Case Study Design}

The current study presents three case studies using a descriptive case study design. The descriptive case study design was chosen in order to provide as much detail about stress reactions as possible, which are rarely discussed in the literature. The study design chosen was selected in order to completely outline and display the timeline and details of events that occurred in these three athletes.

\section{Method of Data Collection}

The data collection procedures were found to be exempt from institutional human subjects review. Medical records were acquired after the athletes provided written informed consent. All data obtained for analysis in the study included diagnostic reports, physician's notes and any certified athletic trainer's notes. Data were collected with help from the medical staff members from Rebound Orthopedics clinic in Portland Oregon and Vancouver Washington or any other part of the Portland State University sports medicine team. 


\section{CHAPTER III}

\section{Case Study \# 1: Tarsal Navicular Stress Reaction}

Tarsal navicular stress reaction is rarely reported in the literature, and there is a need for more research (Burne, Mahoney, Forster, Koehle, Taunton, \& Khan, 2005). One study on tarsal navicular stress fractures in athletes indicated that stress fractures mainly occur in track and field athletes (Khan, Brukner, Kearney, Fuller, J, \& Kiss, 1994). A stress reaction can be thought of in two ways: either a continuum on a scale of stress injuries, or a predisposition to a stress fracture. With the common impact activities of jumping and running in track and field, these would be possible mechanisms of injury based on the thoughts of why stress reactions occur.

\section{Symptoms}

A 22-year old female track and field athlete who participated in the multi-events came into the athletic treatment center complaining of right mid-foot pain. Her history consisted of transferring from a smaller school in a smaller conference. She had been experiencing pain in her right foot for approximately one year. The pain started to affect her ability to practice during winter break when she was training at home, approximately one week before returning to normal practice with her team. This was brought to the attention of her certified athletic trainers (ATCs), who referred her to the team physician, an orthopedic surgeon, and member of the sports medicine team.

During the visit with the physician, she was unable to recall one specific event that brought on her pain, stating it slowly developed over time. According to the medical 
chart notes, the location of pain was isolated to her posterior tibial tendon, with maximum tenderness at the insertion on the navicular bone. Other notes made by the physician include "fairly high arch" and "she does not have a rigid hindfoot". High arches—also known as having a pes cavus foot type-have been determined to be a risk factor for stress fractures (Korpelainen, Orava, Karpakka, Siira, \& Hulkko, 2001; Zeni, Street, Dempsey, \& Staton, 2000; Khan, Brukner, Kearney, Fuller, J, \& Kiss, 1994; Romani, Gieck, Perrin, Saliba, \& Kahler, 2002; McKenzie, Clement, \& Taunton, 1985). She was able to complete some functional tests. There was no obvious swelling noted, and strength and range of motion (ROM) were normal.

The team physician referred her to an orthopedic foot specialist. Additional symptoms were revealed, including aggravation with weight bearing activity, and mild swelling in area of mid tarsus on the medial aspect of her foot. The specialist also found tenderness on palpation in the area of talonavicular and naviculocuneiform joints.

Ten days later she had a follow-up appointment with the original orthopedic surgeon. He found that she was no longer tender over navicular insertion of the tibialis posterior, where the stress reaction occurred. She had been wearing a walking boot, which helped to relieve this pain.

She continued to have pain on the medial aspect of her tarsonavicular joint and had not returned to her normal track and field activities. One month after her initial appointment with the foot specialist, there was a follow-up appointment. She once again experienced tenderness at the insertion of the posterior tibial tendon on the navicular. 
Symptoms remained. The athlete underwent a second set of x-rays and MRI. She was still experiencing mild tenderness at the insertion point of the posterior tibial tendon on the navicular, where she had been tender the entire healing process.

Two weeks into the process of no longer wearing her walking boot symptoms reappeared with tenderness to deep palpation over her navicular. Throughout her healing process, the athlete appeared to continue to have some symptoms, primarily pain on palpation.

\section{Differential Diagnosis}

All of the initial signs and symptoms gave the original impression of posterior tibial tendinitis with navicular irritation. The physician referred her for x-rays and a follow up appointment with a foot specialist to rule out a navicular stress fracture as well. These were the only two diagnosis possibilities throughout the athlete's injury process.

\section{Diagnostic Imaging}

Original X-ray images and the X-rays taken at her first appointment with the foot specialist did not show any evidence of a stress fracture. They did show that the athlete had an accessory navicular. The assessment by the foot specialist yielded a diagnosis of medial midfoot pain of unclear etiology possibly related to stress injury of either the medial cuneiform or tarsonavicular. An MRI was ordered to provide further information. The study confirmed that she had a stress injury on the T2 images of her tarsal navicular. 
One month later the athlete followed up with the foot specialist. X-rays were taken at this appointment and a fracture line was now visible. What had started as a bone stress reaction developed into a bone stress fracture. The specialist stated that this was very subtle and seen best on the lateral view x-ray. The specialist stated that the injury had progressed into a tarsonavicular stress fracture. She was still symptomatic so another MRI was ordered.

Findings of the MRI when compared to her previous MRI showed that navicular minimal dorsal edema was decreased, no significant effusion in the joint, and no mass, edema or abnormal fluid collections were found in the soft tissue. The impression from the MRI report was that navicular of previous stress reaction had been largely resolved, with no acute bony abnormality and no significant tendon abnormality. When the MRI was reviewed with the patient in a follow-up appointment the foot specialist stated that there was evidence of "greatly improved stress reaction in the tarsonavicular" and improvement was evident.

In a follow-up appointment with the foot specialist approximately three weeks after, x-ray images taken, with no evidence of stress fracture.

\section{Treatment/Management Plan}

During her appointment with the foot specialist, the athlete was placed into a walking boot and told to cease track and field activities. The management plan at this time was to continue wearing the walking boot until pain-free and to check in weekly with ATCs in the athletic treatment center. In order to maintain fitness and strength, lowimpact range of motion (ROM) and strengthening activities were prescribed. The 
estimate of time off before returning to track and field activities would be allowed was 46 weeks.

In order to maintain fitness, the athlete performed several activities with her ATC. Pool workouts were one of the major activities, because this allowed her to still do some sport-specific and intense cardiovascular activity, without involving weight bearing. This included lunges, jogging, hurdle walks, marching, hamstring curls, Russian abdominal twists, knee extensions, different types of jumps, full body pull ups, aqua jogging, and swimming using upper body only. In addition to pool workouts, she was allowed to use a stationary bicycle for interval cardiovascular workouts while wearing her walking boot. The athlete was also allowed to use an elliptical trainer. An upper body ergometer was suggested as a part of the maintenance of the athlete's cardiovascular fitness, but it was rarely used.

Ten days later she had a follow-up appointment with the original orthopedic surgeon and he thought her progress was positive and consistent with the proper time frame. He recommended she continue the rest and management plan.

One month later the athlete followed up with the foot specialist; based on the progression of activities, she was told to continue wearing the walking boot and refrain from track and field activities. If he would have seen progression of the fracture line, he then would have had to perform surgery.

Thirteen days later there was notation in the athlete's medical chart notes that an ATC reported there was discussion that the athlete was not compliant with wearing her walking boot. This is important to note here because the athlete's compliance would 
negatively affect her stress reaction and could result in the stress fracture status that was achieved. The specialist also noted that the MRI reviewed would be able to tell whether or not the athlete had been compliant in wearing her walking boot.

The athlete was prescribed custom orthotics which were in the process of being made. Before she was able to receive her orthotics, an arch pad was placed in her walking boot to help alleviate the pain. Once she was asymptomatic she was allowed to progress into activity. The chart notes from the specialist indicated there was a discussion of vitamin D3 and calcium supplementation, as recommended by the surgeon, as well as a discussion of continuously wearing the walking boot (exceptions: sleeping and showering).

After this appointment the athlete saw an orthotic specialist in the athletic treatment center. This process included having her feet analyzed and fitted by the physical therapist (PT) who is part of the sports medicine team. Her feet were placed into neutral position and then casted in order to make the orthotics.

Three weeks later-after an appointment and X-rays—-she was allowed to begin the process of no longer wearing her walking boot. The specialist examined the orthotics she had received, and approved the plan for her to transition out of her cast brace into shoes with orthotics in them. She was allowed to begin weight-bearing activity, primarily walking on a treadmill, and if she remained pain-free, she was allowed to begin light jogging on the treadmill, using the elliptical trainer, and stationary bike without wearing her walking boot. 
Two-weeks after progressing into light activity that included weight-bearing, she had a follow-up with the foot specialist. She was still tender to deep palpation over her navicular. At this point the physician assessed that her tarsonavicular stress fracture had healed and she could continue to progress into full impact activities over the next six weeks.

At her six week check-up with the foot specialist, the athlete was still having some symptoms in her posterior tibial tendon. She had no swelling, but mild tenderness on her posterior tibial tendon from her ankle to the navicular tuberosity. The assessment at this time demonstrated that she now had a mild case of posterior tibial tendinitis. The athlete continued her current strengthening program and was instructed to have a followup appointment with the physician prior to her next season's training. She stated at the time that her orthotics were helpful, so the specialist advised her to continue using them. At this point she was fully released to continue with her track and field activities.

\section{Post Return to Activity}

Upon returning to school and training with the track and field team in the fall, there was no notation by the ATC or other medical staff that the athlete experienced problems with the previous stress injury until after the indoor season of track and field competition. It is important to note this in the study, because it is known that stress injuries, whether stress fracture or stress reaction can reoccur. A little over a year after the initial symptoms and a stress reaction to the tarsal navicular, she was having increasing pain during indoor track season, which felt similar to the symptoms she had 
with her previous stress injury. There was also notable edema in her foot, and pain in her tarsal navicular region.

She was referred to the same foot specialist who had treated her for the previous injury. The only notable symptom in the chart note was minimal swelling. X-rays were obtained at the time of the appointment and reviewed with the athlete. The x-rays did not reveal any ongoing navicular stress fracture or a stress reaction, and appeared to be normal. The diagnosis given at the time was irritation at insertion of the posterior tibialis. The physician suggested following-up with an MRI. This is the last season for her to compete in, as she is a fifth year senior. Therefore, she will modify training as needed and follow-up, if necessary, after her competitive season has concluded.

This case study was similar to information in the literature about stress injuries. The athlete had follow-up symptoms which could have been another stress reaction. Reoccurrence does happen, and the two articles specifically about the tarsal navicular indicate that subjects remained symptomatic, or another stress injury did occur (Burne, Mahoney, Forster, Koehle, Taunton, \& Khan, 2005; Khan, Brukner, Kearney, Fuller, Bradshaw, \& Kiss, 1994).

\section{Case Study\# 2: Stress Reaction of the Tibia}

Runners have an increased risk of stress injuries, with the tibia being the most common bone affected (Fredericson, Bergman, Hoffman, \& Dillingham, 1995; Kiuru, Pihlajamaki, \& Ahovuo, 2004). Freshmen commonly experience stress injuries (Fredericson, Bergman, Hoffman, \& Dillingham, 1995). The case reported here is about a freshman female who experienced a tibial stress reaction. She is a cross country runner, 
meaning that she participated in three competitive seasons: cross-country in the fall and track and field indoor and outdoor seasons. She competed in distance running events for the track and field seasons.

\section{Symptoms}

An 18-year old cross-country and track and field athlete presented with pain in the middle third of her right tibia. The initial injury evaluation was performed by the graduate assistant certified athletic trainer (GA ATC). Pain was increased while running and the athlete indicated that it hurt constantly, even when simply walking. There was a notable slight limp in her gait. The athlete's tibia did not have an obvious deformity, ecchymosis (discoloration) or edema (swelling) present. Her strength was normal, but she had increased pain during ankle eversion. When she was weight bearing she had pain as well. The assessment at this time was possible posterior tibial inflammation or a stress injury. There was a discussion about what to do next, with the upcoming cross-country conference championships being a consideration. She saw the team physician and orthopedic surgeon soon after this time and he found this to be an overuse injury that would resolve with rest. There was discussion between the athlete, coach and GA ATC concluding the plan was to allow the athlete to finish out the season and then check in again with the team orthopedist.

After the cross-country season was finished, the athlete was again seen by the team physician, the orthopedic surgeon. The chief complaint was her right leg, but she had pain in both feet and her right leg. She had taken an initial rest period and decreased loading, so she finished the cross-country season and stated her foot pain had resolved, 
with continuing leg pain. On physical examination her primary symptom was tenderness along posteromedial border of her tibia at the junction of the middle and distal thirds of the tibia.

\section{Differential Diagnosis}

Including the initial ATC note, posterior tibial inflammation or tendinitis were injury possibilities. Shin splints were given as an impression by the radiology report and stated to be ruled out by the team physician. None of these were the final diagnosis.

\section{Diagnostic Imaging}

X-rays and MRI of the right tibia and fibula were requested by the team physician/surgeon at the first visit after cross-country season had finished. Findings of the MRI included medullary edema, minimal cortical edema, surrounding posterior medial and medial periosteal fluid, just below midpoint of the tibia with bony abnormality length being $3.2 \mathrm{~cm}$. The radiologist noted on the MRI report that this appeared to be a stress reaction of the distal tibia or shin splints.

When the team physician met with the athlete after the MRI and x-rays were obtained, he stated that the MRI showed "quite a severe stress reaction along the medial tibia with marrow edema and soft tissue edema about the tibia". There was no frank break in cortex of the bone, as neither the MRI nor x-ray revealed such evidence. 


\section{Diagnosis}

After diagnostic imaging, the impression and diagnosis given by the team physician was a severe stress reaction in medial tibia.

\section{Treatment/Management/Immobilization Plan}

The initial plan after diagnosis was to unload the tibia for six weeks. During this time she was allowed to maintain cardiovascular fitness via walking, riding a stationary bike, or using the elliptical trainer. No jumping or running was allowed until the athlete was pain free. After six weeks, she was scheduled for a follow-up appointment with the team physician. The athlete was also placed in a walking boot because she was unable to walk pain-free.

The training plan for the athlete was managed with pool workouts and stationary bike workouts with the GA ATC and the cross-country coach. This allowed her to maintain her fitness level while not participating in impact activities.

\section{Returning to Activity}

A follow-up appointment with the team physician after the recommended sixweek period of rest revealed the athlete had periods of no pain. The physical examination revealed some firmness where the stress reaction occurred on her tibia, medially at the junction of the mid and distal thirds, but she experienced minimal tenderness. At the appointment the team physician allowed her to discontinue wearing the boot and advance to some light jogging five days after the appointment was made, which would be the six week point in her management plan. The return to activity plan as advised by the team 
physician was to advance from walking weight bearing to jogging approximately 10 percent per week. Her full release to participate in full workouts was approximately two months from the day she began jogging. Custom orthotics were prescribed by the physician to decrease pronation in her feet and ensure that the running shoes she used were adequate.

At this point, the athlete was given a return-to-activity management plan. Custom orthotics were ordered by a PT that makes orthotics for the sports medicine team utilizing the Biomechanical Services orthotics company.

\section{Post Return to Activity}

Approximately three months after her initial return to activity, the athlete began feeling symptoms of medial foot pain. She was referred by the ATC staff to meet with the physician's assistant (PA) who works closely with the team physician. He did not find any tenderness in her navicular area or tenderness in the medial talus area. He also found full active and passive ROM with full strength in her foot. He was unable to find anything significantly wrong with her foot and suggested continuing to treat conservatively with taping of the arches, therapeutic modalities, and stretching. If symptoms did not get progressively better, the PA agreed she would return and a further work-up would be done with diagnostic imaging including an MRI to rule out a stress fracture or a stress reaction in medial cuneiform or navicular area.

It was important that this athlete was referred for $\mathrm{x}$-rays and followed-up with the orthopedic clinic, because athletes who have previous stress injuries are typically more 
prone to subsequent stress injury (Kiuru, Pihlajamaki, \& Ahovuo, 2004). This athlete should be monitored throughout the rest of her career as well.

\section{Case Study \# 3: Stress Reaction in the Foot}

The foot is a common site for stress injuries, with the second or third metatarsal as the most common bones cited (Kiuru, Pihlajamaki, \& Ahovuo, 2004). The following is not a typical stress reaction, and although it may not be considered a true stress injury, it can be defended as a stress reaction because of the symptoms and history of the athlete.

A 19-year old female cross-country runner and distance runner on the track and field team was practicing under direction of her coach, following a workout plan he developed for his athletes during their winter break, when she began experiencing pain in her left foot. She contacted her ATC, explained her symptoms, and was instructed by the ATC to immediately discontinue running. The athlete followed up with the ATC in the athletic treatment center on campus as soon as winter break was over.

\section{Symptoms}

Initial symptoms reported to the ATC were pain over the second metatarsal (MT) head, and pain with flexion and extension of the second toe. Both tap test and squeeze test were negative. At her follow-up appointment with the general team physician a few days later, her symptoms included pain with walking, but there was no ecchymosis or deformity present. A history of the athlete during the clinical exam revealed that she had a similar problem in high school. The athlete was a freshman so she had an increase in her level and distance of running with practices and training in cross-country and track at 
the college level within the past year. After meeting with the team orthopedic surgeon, her symptoms were the same. She had taken two weeks off before returning to running when she came back to school after winter break, and developed pain again a few days later. She had normal weight and body habitus, was eumenorrheic, had no history of stress injuries, and no history of metabolic disease. The team physician also noted she had a normal gait, no swelling in her foot, although she experienced tenderness at the second MT neck, on both the dorsal and plantar aspects of her foot.

\section{Differential Diagnosis}

Based on the location of pain, a Morton's Neuroma was suspected initially, or an intermetatarsal neuroma. A Morton's Neuroma or intermetatarsal neuroma, means there is entrapment of the nerve in the foot, that can create radiating pain through the foot. When the pain did subside with treatment for a neuroma, and when the athlete visited the general team physician this was no longer the suspicion. The general team physician felt that there was left foot second metatarsal pain with suspicion of a stress fracture. After referral to the team orthopedic surgeon, he also thought that a stress fracture was possible.

\section{Diagnostic Imaging}

When meeting with the team orthopedic surgeon, x-ray images taken did not show a fracture line. An MRI was ordered to provide further information. Based on the radiologist's report, findings included no metatarsal bone marrow edema to suggest a stress fracture, but diffuse marrow edema incidentally noted within partially visualized great toe distal phalanx. Biomechanical stress was considered as the cause. There was no 
significant subcutaneous or muscular edema in area of clinical suspicion, no abnormal soft tissue mass, no intermetatarsal neuroma, and no gross metatarsophalangeal joint collateral ligament injury in area of clinical suspicion. When the team orthopedic surgeon reviewed the MRI, he indicated there was no evidence of stress fracture or other significant abnormality in the area of the second metatarsal neck, where the athlete was experiencing symptoms. There was, however, some diffuse marrow edema seen in distal phalanx of the great toe, but the patient was asymptomatic in that area.

\section{Diagnosis}

There was no official diagnosis given, but as there was marrow edema found in the distal phalanx of the great toe, this could be considered to be a stress reaction based on the grading scale of stress reactions, as seen in the literature. Based on a classification scale of stress fractures and reactions presented by Jones, Harris, Vinh and Rubin (1989), this would be a grade I or II (mild to moderate) stress reaction, because she was having symptoms but there was no palpable mass. There was no bone scan conducted, so it is difficult to identify any changes in bone. While there were no noticeable changes in the MRI in the suspected area of the second metatarsal, the edema around the distal phalanx of the big toe does indicate that there was some change in the foot causing pain.

\section{Management Plan}

The athlete was traveling to a track meet the day following her x-ray, so she was allowed to run the $3 \mathrm{~K}$ if she was pain free, because there was no fracture line. This was before the discovery in her MRI. She completed the race. It was suggested that she get 
custom orthotics. The team physician recommended a full length orthotic to unload the second metatarsal head.

After a discussion between the ATC, coach, and athlete, it was decided that rest would be involved in the plan in order for the athlete to continue participating in the indoor track season. Padding, orthotics, and any therapeutic modalities that would help with pain were utilized, and eventually the athlete was pain-free and able to return to track and field activities as normal. The athlete was able to finish her indoor track season pain-free.

\section{Post Return to Activity}

This athlete had recurring foot pain when she had to be on crutches, using her left foot only. There was no further work-up, because she was already resting due to the fact that she sustained a stress fracture to her sacrum. She should also be continuously monitored for stress injuries throughout her collegiate career, due to the fact that she maintained a stress injury during her first year at the college level. 


\section{CHAPTER IV}

\section{Literature Review}

\section{History}

Stress injuries to the bone were first noted by a Prussian military surgeon, Briethaupt, in 1855 when he described swelling in the feet of soldiers (Kiuru, Pihlajamaki, \& Ahovuo, 2004; Bennell, Malcolm, Wark, \& Brukner, 1996; Sterling, Edelstein, Calvo, \& Webb II, 1992; Jones, Harris, Vinh, \& Rubin, 1989). When radiographs were invented 42 years later, signs and symptoms were able to be attributed to stress fractures in the metatarsal (MT) bones (Bennell, Malcolm, Wark, \& Brukner, 1996). These were first found mainly in military recruits. Then, in 1921, civilians were found to have stress fractures, noted in female civilians in 1921 by Deutschlander (Bennell, Malcolm, Wark, \& Brukner, 1996). In 1932 there was more evidence of what is now called a "march fracture", showing evidence locally of fracture healing and callus formation in a publication by Strauss (Jones, Harris, Vinh, \& Rubin, 1989). March fractures were named due to the amount of marching in the military, thus causing the fracture. The first explanation concerning the cause of stress fractures occurred in 1937 by Detlefsen and by Hartley in 1942 when they stated stress fractures are related to bone exhaustion, as occurs in common metal fatigue (Bennell, Malcolm, Wark, \& Brukner, 1996). It was in the 1970s that bone scintigraphy, or bone scan, became an important imaging tool in the detection of bone stress injuries (Kiuru, Pihlajamaki, \& Ahovuo, 2004). Sports medicine practices were estimating the occurrence of stress fractures at about $10 \%$ of injuries by the 1980s (Jones, Harris, Vinh, \& Rubin, 1989). This is still the 
perceived percentage of commonality seen by sports medicine clinics today (Fredericson, Bergman, Hoffman, \& Dillingham, 1995; Kiuru, Pihlajamaki, \& Ahovuo, 2004; Sterling, Edelstein, Calvo, \& Webb II, 1992). This means that approximately 1 of every 10 athletes experience a stress injury.

The first description of a tarsal navicular stress fracture was in 1970, which was described as rare (Khan, Brukner, Kearney, Fuller, Bradshaw, \& Kiss, 1994). Tarsal navicular stress fracture specifically was first described in imaging tests and clinical outcomes by Joseph Torg and colleagues in approximately 1985 (Burne, Mahoney, Forster, Koehle, Taunton, \& Khan, 2005). The etiology is still evolving today with advances in diagnostic imaging and treatment of bone stress injuries, including terminology to clarify the differential diagnosis of a true stress fracture versus a stress reaction (Jones, Harris, Vinh, \& Rubin, 1989). Looking into the history of an injury allows one to see how far the current knowledge of the injury has come.

\section{Anatomy of Bone}

In order to understand the development of a stress fracture or stress reaction to a bone, there needs to be an understanding of bony anatomy. Long bones are the bones in limbs, such as the tibia and fibula in the lower leg (Marieb \& Hoehn, 2007). They consist of an outer lining of periosteum, then compact bone surrounding yellow bone marrow that is lined with endosteum (Marieb \& Hoehn, 2007). The diaphysis is the tubular shaft of the bone, with the epiphyses at each end (Marieb \& Hoehn, 2007).

Bone is a dynamic tissue, meaning that once formed, it continually undergoes remodeling and responds to stresses and strains placed on it (Kiuru, Pihlajamaki, \& 
Ahovuo, 2004). There are two forms of bone: cortical and trabecular (Zeni, Street, Dempsey, \& Staton, 2000). Cortical bone is compact bone surrounding the marrow in the shaft of the bone. Trabecular bone is also known as spongy or cancellous bone surrounds marrow spaces adjacent to the cortical bone and makes up the majority of the bone. Lamellar bone is the only type relevant to stress injuries, as it is found in individuals four years or older (Zeni, Street, Dempsey, \& Staton, 2000). There are three basic bone cells: osteoclasts, osteoblasts, and osteocytes. Osteoclasts are involved in resorbing or breaking down the bone matrix (Marieb \& Hoehn, 2007). Osteoblasts are involved in bone formation (Marieb \& Hoehn, 2007) and line the surface of the bones deep to the periosteum (Zeni, Street, Dempsey, \& Staton, 2000). An osteoblast is stimulated by hormones and external stresses defined by Wolff's Law (Zeni, Street, Dempsey, \& Staton, 2000). Osteocytes are mature bone cells (Marieb \& Hoehn, 2007), which maintain bone (Zeni, Street, Dempsey, \& Staton, 2000) and hydroxyapatite (an inorganic calcium phosphate matrix) . Central canals, also called haversian canals, run through the core of each osteon. An osteon is the basic structural unit, consisting of the central—or haversian—canal network; these canals contain blood vessels and nerves to support the osteon's cells (Marieb \& Hoehn, 2007).

Bone composition is different for each person depending on age, diet, disease, genetics and location in the body (Zeni, Street, Dempsey, \& Staton, 2000). Peak bone mass is reached by about age 25 or 30 (Zeni, Street, Dempsey, \& Staton, 2000), so it is important that up until this age the body is supplied with enough nutrients to acquire bone mass and strength. Athletes need to be educated and understand they can be acquiring enough calcium and other nutrients in their diet to maximize their peak bone mass. 
Wolff's Law was introduced in a series of articles between 1869 and 1892, by Julius Wolff (Jones, Harris, Vinh, \& Rubin, 1989). In stress injuries, there is a process of bone adaptation resulting from alterations in the mechanical environment (Jones, Harris, Vinh, \& Rubin, 1989). Bone stress can strengthen bone, but high levels of stress can cause remodeling to become too accelerated, resulting in fatigue damage (Rome, Handoll, \& Ashford, 2009). When bone is abnormal unable to properly adapt to the normal stress placed upon it, stress injuries occur (Kiuru, Pihlajamaki, \& Ahovuo, 2004). Along with intense training of athletes, it is possible that this results in microarchitectural damage at bone sites that are maximally stressed (Bennell, Malcolm, Wark, \& Brukner, 1996). There is a balance in the process of bone adaptation to new stresses. As this balance becomes more disrupted, resorption occurs before new bone can be incorporated (Jones, Harris, Vinh, \& Rubin, 1989). At this time remodeling is hampered and the risk for stress injury increases(Jones, Harris, Vinh, \& Rubin, 1989).

Bone remodeling is thought to repair bone by either directed repair, or simple random remodeling (Bennell, Malcolm, Wark, \& Brukner, 1996). Directed repair means the remodeling units are directed to the location of damage, whereas simple random remodeling refers to units keeping up with the damage accumulation by continual breakdown and buildup of bone (Bennell, Malcolm, Wark, \& Brukner, 1996). Once remodeling has begun, central or haversian canal formation and osteoblast support with lamellar bone begins, usually within ten to fourteen days (Romani, Gieck, Perrin, Saliba, \& Kahler, 2002). Then lamellar bones begin to be converted by mature osteocytes (Romani, Gieck, Perrin, Saliba, \& Kahler, 2002). This process lags behind resorption, which can lead to a temporarily weakened bone, due to a hollow central canal (Romani, 
Gieck, Perrin, Saliba, \& Kahler, 2002). This results in bringing about a "weak third week", thought to be when a stress fracture is most likely to develop (Romani, Gieck, Perrin, Saliba, \& Kahler, 2002). This is why a training regimen planned around this three-week system has been suggested when an athlete is exposed to a new activity or an increase in current activity (i.e., more intense training for two weeks followed by less intense training in the third week) (Romani, Gieck, Perrin, Saliba, \& Kahler, 2002). When remodeling is accelerated or depressed, stress fractures may develop in an individual who is intensely training. This is applicable to athletes, particularly track athletes who have multiple seasons with periods of intense training such as distance running or the repetitive jumping and sprinting performed by a multi-event athlete.

\section{Risk Factors}

There are numerous risk factors that can predispose an individual to a stress injury. These factors can be classified as intrinsic and extrinsic factors. The extrinsic risk factors consist of things outside of the body that can predispose an individual to stress injuries. Intrinsic factors apply to sources within the body that can increase the risk of stress injuries. Many of these risk factors have not been studied specifically, and much remains to be elucidated concerning their relationships to stress injuries. If a risk factor can be modified in an effort to prevent stress injuries, then it should be utilized.

A study looking at recurring stress fractures in athletes identified several risk factors. High weekly training mileage for runners was one of them (Korpelainen, Orava, Karpakka, Siira, \& Hulkko, 2001). Biomechanical factors_-an example of intrinsic risk factors-were measured and analyzed in the participants, and included narrow tibia, high 
degree of hip external rotation, varus alignment in the ankle and forefoot, an ankle that hyperpronates, when the longitudinal arch of the foot is high, and leg length discrepancy (Korpelainen, Orava, Karpakka, Siira, \& Hulkko, 2001). In this study, significant risk factors were leg length discrepancy, high weekly mileage, high longitudinal arch, and forefoot varus (Korpelainen, Orava, Karpakka, Siira, \& Hulkko, 2001). For stress injuries to the foot, a biomechanical risk factor mentioned in a separate study was a short first metatarsal and metatarsus adductus (Khan, Brukner, Kearney, Fuller, Bradshaw, \& Kiss, 1994). Other biomechanical risk factors examined in a review study included anteversion of the femur, varus or valgus knees, tibia vara, varus or valgus calcaneous, and flat foot; all were found to increase the risk of stress injuries (Lassus, Tulikoura, Konttinen, Salo, \& Santavirta, 2002). These intrinsic risk factors certainly provide clues that could be utilized in prevention efforts.

Additional intrinsic factors were also discussed in the same review; however, they were relevant only to females. Menstruation, menstrual disturbances and delayed menarche were identified as risk factors (Lassus, Tulikoura, Konttinen, Salo, \& Santavirta, 2002; Kelsey et al., 2007; Zeni, Street, Dempsey, \& Staton, 2000). Age at first menarche has been found to relate to stress injuries, and although there is no proven link, female athletes tend to reach menarche later than non-athletes (Zeni, Street, Dempsey, \& Staton, 2000). Contraceptives have sometimes been found to decrease risk of stress injuries and estrogen replacement therapy has been hypothesized to reduce stress injuries, but neither of these were made with supporting research behind them (Lassus, Tulikoura, Konttinen, Salo, \& Santavirta, 2002). A separate study of risk factors in females concluded that oral contraceptive pills seem to have a protective effect (Zeni, 
Street, Dempsey, \& Staton, 2000). This suggests there is a hormonal influence that plays a role in the occurrence of stress injuries.

These additional risk factors for females put them at a greater risk for stress injuries than males (Kiuru, Pihlajamaki, \& Ahovuo, 2004; Zeni, Street, Dempsey, \& Staton, 2000; Sterling, Edelstein, Calvo, \& Webb II, 1992; Jones, Harris, Vinh, \& Rubin, 1989). This is especially true if a female is suffering from the female athlete triad (Kiuru, Pihlajamaki, \& Ahovuo, 2004). The female athlete triad consists of excessive physical activity, disordered eating, and amenorrhea which can result in loss of bone density (osteopenia), which may result in osteoporosis (Zeni, Street, Dempsey, \& Staton, 2000). Eating disorders are also a risk factor for stress injuries (Zeni, Street, Dempsey, \& Staton, 2000). Disordered eating — specifically anorexia nervosa — puts athletes at a higher risk for developing stress injuries due to low bone mineral density (BMD) (Zeni, Street, Dempsey, \& Staton, 2000).

Bone mineral density or bone mass is important, as young females with low bone mass are at greater risk for stress injuries (Kelsey et al., 2007). When bone loading is increased in accordance with the intense training of athletics, a higher BMD is needed as well, conversely, the risk of stress fractures is increased with a lower BMD, although more studies are needed (Kelsey et al., 2007). A study focusing on risk factors for young female cross-country athletes stated that for each standard deviation decrease in wholebody mineral, stress fracture risk rate increased almost twofold (Kelsey et al., 2007).

Other extrinsic risk factors not yet mentioned are training regimen, footwear, and training surface (Zeni, Street, Dempsey, \& Staton, 2000). High running mileage 
increases the risk of stress injuries, however, any abrupt change in duration, frequency or intensity of an athlete's training regimen may also affect the risk of stress injuries (Zeni, Street, Dempsey, \& Staton, 2000). Shoe age, or wear, is a risk factor in relation to shock absorbency (Zeni, Street, Dempsey, \& Staton, 2000). Surfaces on which an athlete trains can increase the risk of stress injury if the surface is uneven, presumably because muscle fatigue puts more stress on bone (Zeni, Street, Dempsey, \& Staton, 2000).

Other intrinsic factors include race, bone geometry and foot structure (Zeni, Street, Dempsey, \& Staton, 2000). Caucasian females, along with Asian females are found to be at a significantly higher risk than African-American, possibly due to bone turnover or peak bone density (Zeni, Street, Dempsey, \& Staton, 2000). The geometry of bone relates to stress injury due to the fact that, theoretically, lower cross-sectional areas in bone along with moments of inertia are not as able to withstand external loading forces (Zeni, Street, Dempsey, \& Staton, 2000). It is also hypothesized that narrower bones in women contribute to their higher risk of stress injuries (Zeni, Street, Dempsey, \& Staton, 2000). With a foot structure such as a high-arched foot, or pes cavus foot, more force is transmitted to the tibia and femur, putting these bones at increased risk for stress injury (Zeni, Street, Dempsey, \& Staton, 2000). Conversely, a more flat-foot structure absorbs more force and less is transmitted to the other bones in the leg, creating risk for stress injury to these bones (Zeni, Street, Dempsey, \& Staton, 2000).

\section{Symptoms}

When pain related to stress reactions first begins, it is gradual and typically only felt by the athlete when active, and dependent on the stress load experienced that day 
(Lassus, Tulikoura, Konttinen, Salo, \& Santavirta, 2002; Kiuru, Pihlajamaki, \& Ahovuo, 2004). The pain eventually progresses to the point that it can be felt at rest, and night pain is common as well (Lassus, Tulikoura, Konttinen, Salo, \& Santavirta, 2002; Kelsey et al., 2007). Pain usually begins in a localized area and may never progress to any other region, although soft tissues surrounding the bone may experience swelling (Lassus, Tulikoura, Konttinen, Salo, \& Santavirta, 2002). If symptoms continue to progress it is possible that percussion, either direct or indirect, may elicit pain (Lassus, Tulikoura, Konttinen, Salo, \& Santavirta, 2002; Fredericson, Bergman, Hoffman, \& Dillingham, 1995). The athlete may be unable to recall a single event that created the pain which is associated with stress injuries (i.e., an insidious onset) (Fredericson, Bergman, Hoffman, \& Dillingham, 1995). Pain can either be acute or chronic (Kelsey et al., 2007). Along with pain, the athlete may have a limp, pitting edema, and redness or warmth near the area of the stress injury with a palpable protuberance at the site (Kelsey et al., 2007).

Specifically discussing symptoms in tarsal navicular stress injuries, there is typically pain or tenderness over the dorsal proximal navicular bone, which can also radiate along the medial part of the longitudinal arch in the foot or on the dorsum aspect of the foot (Khan, Brukner, Kearney, Fuller, Bradshaw, \& Kiss, 1994). The athlete will still have normal range of motion and strength in her ankle (Khan, Brukner, Kearney, Fuller, Bradshaw, \& Kiss, 1994).

\section{Diagnostic Imaging}

Along with gaining an extensive history and information about all symptoms presented by the athlete, diagnostic imaging is crucial in aiding the diagnosis of stress 
injuries, especially differentiating between the grades of stress reactions (Lassus, Tulikoura, Konttinen, Salo, \& Santavirta, 2002) or between a stress fracture and stress reaction. In tarsal navicular stress injuries, MRI is more sensitive in diagnosing a navicular stress fracture or reaction than CT or x-ray images (Burne, Mahoney, Forster, Koehle, Taunton, \& Khan, 2005).

Magnetic resonance imaging (MRI) and computed tomography (CT) scan have their respective strengths and weaknesses in their use. One positive aspect of a CT scan is that it may reveal a disruption of cortical bone (Burne, Mahoney, Forster, Koehle, Taunton, \& Khan, 2005). On the other hand, it is unable to determine if there are any disruptions in fine trabeculae (i.e., is unable to detect the minute details) (Burne, Mahoney, Forster, Koehle, Taunton, \& Khan, 2005). Similarly, an MRI is unable to detect disruption of trabeculae; however, it has superior contrast resolution and can therefore reveal any presence of edema, blood, or fibrous tissue that would possibly be in the fracture line (Burne, Mahoney, Forster, Koehle, Taunton, \& Khan, 2005). An MRI is sensitive and more specific than scintigraphy (also known as a bone scan), and can provide information about the soft tissues around the bone in all three dimensions (Lassus, Tulikoura, Konttinen, Salo, \& Santavirta, 2002). It is most accurate in the first three weeks, and is better for early detection of stress injuries and differential diagnosis (Lassus, Tulikoura, Konttinen, Salo, \& Santavirta, 2002). An MRI is specific in that it can show changes in the bone marrow (Kiuru, Pihlajamaki, \& Ahovuo, 2004). This is important because a typical initial sign of stress injury is endosteal marrow edema (Kiuru, Pihlajamaki, \& Ahovuo, 2004). 
Both an MRI and a CT scan are better than standard radiography at detecting stress injuries. MRIs have been gaining popularity recently due to higher resolution and less exposure to x-rays (Kiuru, Pihlajamaki, \& Ahovuo, 2004). One study suggested that a CT scan is of lower value diagnostically than an MRI, unless there is a longitudinal stress fracture of the tibia (Kiuru, Pihlajamaki, \& Ahovuo, 2004). However, it is important to remember that in order of diagnostic imaging, an x-ray should be the first imaging modality, and an MRI or a CT scan next if more information is needed (Kiuru, Pihlajamaki, \& Ahovuo, 2004). It is possible there is a fracture line visible in clear radiographs, depending on the view (Kiuru, Pihlajamaki, \& Ahovuo, 2004).

Additionally, new periosteal bone formation may be seen with a callus formation in x-ray images (Kiuru, Pihlajamaki, \& Ahovuo, 2004).

\section{Graded Stress Injury Scale}

It is important to include crucial information about stress injuries, and one piece of information vital to this literature review is an article titled Exercise-Induced Stress Fractures and Stress Reactions of Bone: Epidemiology, Etiology, and Classification (Jones, Harris, Vinh, \& Rubin, 1989). This article addresses classification and grading of stress fractures and reactions (Jones, Harris, Vinh, \& Rubin, 1989). The authors disagree with the general term "stress fracture" that has been used in the past to describe what was most likely a stress reaction (Jones, Harris, Vinh, \& Rubin, 1989).

The first grade classification is "grade 0", meaning that there is physiologic response of bone to change due to a load or repeated loads (Jones, Harris, Vinh, \& Rubin, 1989). Bone scan would be positive, but most likely the athlete would be non- 
symptomatic and there is no immediate danger to the bone and no detection from x-rays (Jones, Harris, Vinh, \& Rubin, 1989). A "grade 0" classification could also be referred to as normal remodeling (Jones, Harris, Vinh, \& Rubin, 1989). A "grade I" classification refers to a clinically significant stress reaction of bone, meaning there are symptoms (e.g., local pain increased with activity or a recent change in activity) with minimal tenderness (Jones, Harris, Vinh, \& Rubin, 1989). A bone scan on grade I would be positive, however x-rays will still be undetectable (Jones, Harris, Vinh, \& Rubin, 1989). This could be called a mild stress reaction (Jones, Harris, Vinh, \& Rubin, 1989). A "grade II" classification on the scale would denote a clinically significant stress reaction, a positive bone scan, and barely detectable changes with x-rays (Jones, Harris, Vinh, \& Rubin, 1989). Symptoms would include pain locally, mild tenderness, however, with no palpable mass and an onset of pain due to change in activity, a "grade II" classification could be referred to as a moderate stress reaction (Jones, Harris, Vinh, \& Rubin, 1989).

A "grade III" classification includes stress reactions with potential structural significance with bone scans showing this change, as well as x-rays (Jones, Harris, Vinh, \& Rubin, 1989). Symptoms are present in the athlete as well (Jones, Harris, Vinh, \& Rubin, 1989). There are lesions with local pain that do not always cease with rest, local tenderness and there may be a palpable mass (Jones, Harris, Vinh, \& Rubin, 1989). There is usually a history of increased activity recently, insidious onset of pain, and an increase in the level and duration of discomfort if the activity were to continue (Jones, Harris, Vinh, \& Rubin, 1989). This could be labeled as a severe stress reaction (Jones, Harris, Vinh, \& Rubin, 1989). A "grade IV" classification could be referred to as a stress fracture, meaning the bone failed structurally and there is a frank fracture or break 
in the continuity of the bone (Jones, Harris, Vinh, \& Rubin, 1989). X-rays would reveal this frank fracture and evidence of a chronic remodeling process and new bone formation, rather than one single event of bone overloading (Jones, Harris, Vinh, \& Rubin, 1989). Extreme pain is accompanied with a stress fracture, possibly making the most minimal weight bearing difficult (Jones, Harris, Vinh, \& Rubin, 1989). Other symptoms are similar to the other characteristics of the other grades (Jones, Harris, Vinh, \& Rubin, 1989).

\section{Treatment}

After a complete work-up (i.e., symptoms presented, imaging performed, and a diagnosis of stress injury), treatment may include an immobilization plan regarding the amount of rest or unloading needed, and an exercise prescription for maintaining the athlete's cardiovascular fitness. These should be completed prior to allowing the athlete to begin a return to activity program. The primary concern is the prevention of stress injuries. The next crucial step in treatment is ceasing physical activity that aggravates symptoms (Kelsey et al., 2007). This typically means non-impact or weight bearing activities. Early diagnosis is also important, as is eliminating or reducing bone load (Lassus, Tulikoura, Konttinen, Salo, \& Santavirta, 2002). Therapeutic modalities that are helpful when used in the treatment of stress injuries include: cold treatments, phonophoresis (using ultrasound with medication such as a topical NSAID), electric and electromagnetic treatments, and pulsed low intensity ultrasound (Lassus, Tulikoura, Konttinen, Salo, \& Santavirta, 2002). Acupuncture is also a treatment option 
(Fredericson, Bergman, Hoffman, \& Dillingham, 1995). The overall goal is to reduce the amount of pain experienced by the athlete.

The guideline for treatment is pain reported by the athlete, and the goals of the active rest period of the athlete have been stated in an article based on the acronym REST: removal of abnormal stress, exercise to maintain fitness and muscle mass, safe and pain free return to activity level, time for bone maturity to catch up with an increased bone remodeling (Romani, Gieck, Perrin, Saliba, \& Kahler, 2002). A three phase process to take advantage of the physiologic healing cycle of bone, while ensuring pain is managed during the healing process has been described by Romani et al. (2002). This is, once again, a two week period where stresses can be applied normally or more intense activities can occur, followed by a lighter "rest week". Other treatment modality options include: transcutaneous electrical nerve stimulation (TENS) or high volt electrical stimulation (e-stim), ice massage, contrast bath, and ultrasound (Romani, Gieck, Perrin, Saliba, \& Kahler, 2002). Rehabilitation exercises included are: towel toe curls, ankle isometrics, sitting range of motion on a wobble or biomechanical ankle platform system (BAPS) board (Romani, Gieck, Perrin, Saliba, \& Kahler, 2002).

A separate study discussed and recommended Clement's 2-stage stress fracture management program (Sterling, Edelstein, Calvo, \& Webb II, 1992). Decreasing pain is the goal of the first stage which includes: NSAIDs, PT, ice massage, modified rest or weight bearing exercise program, avoiding the activity that causes pain, stretching and flexibility, muscle strengthening and fitness alternatives (Sterling, Edelstein, Calvo, \& Webb II, 1992). The second stage is to be pain free for 10 to 14 days in the first stage 
and then begin reintroducing the offending mechanism with an every other day rest. Pain is the guide to progress, which means that it may be necessary to alter equipment and biomechanics in the training program as necessary (Sterling, Edelstein, Calvo, \& Webb II, 1992). Clement 2-stage stress fracture management program is a good sum of overall goals and a guide of a rehabilitation and treatment program.

When a tarsal navicular stress injury is diagnosed, there is an outstanding recommendation of six weeks non weightbearing cast immobilization as stated in a study that cited an Australian report with support from CT images (Burne, Mahoney, Forster, Koehle, Taunton, \& Khan, 2005). It is important to note that there is no set period of time an athlete will be prohibited from participating in impact or weight bearing activities. It is largely dependent on the grade of stress reaction or stress fracture. A study focusing on tibial stress reaction in runners discovered that an athlete suffering from a grade 1 stress injury could return to running on grass or soft surface in about 2 to 3 weeks, while a grade 4 stress fracture required wearing a cast for 6 weeks with another 6 weeks of non-impact activity (Fredericson, Bergman, Hoffman, \& Dillingham, 1995). The physician plays an important role on how much time an athlete will be withheld from participating in his or her sport, and decisions depend on whether the physician treats the stress injury conservatively or not.

Non-steroidal anti-inflammatory drugs (NSAIDs) have not been shown to improve or affect healing, however, they can be recommended for treatment for pain and edema in the early phase of a stress injury (Lassus, Tulikoura, Konttinen, Salo, \& Santavirta, 2002). If there is a stress injury in the fibula, a long air-cast could be helpful 
(Lassus, Tulikoura, Konttinen, Salo, \& Santavirta, 2002). Any foot or tibial stress injury may benefit from a walking cast brace boot to relieve the pain during activities of daily living (ADLs). If pain is still present while wearing a boot, then crutches may be utilized as well. Surgery is typically only indicated in the case of a true stress fracture in the tarsal navicular.

While the athlete may not be able to bear weight or participate in impact activities, he or she can still maintain cardiovascular fitness by other means. Crosstraining is vital during the rehabilitation and treatment period, along with flexibility and muscle strengthening (Fredericson, Bergman, Hoffman, \& Dillingham, 1995). Stationary cycling, aqua jogging, swimming or water treading and upper-body ergometer are helpful in maintaining aerobic fitness (Romani, Gieck, Perrin, Saliba, \& Kahler, 2002). An important consideration is that this cross-training activity does not irritate the stress injury.

Prevention of any injury is the goal of the sports medicine staff and deserves mention here. Avoiding sudden changes in running track surface and shoes, and avoiding overtraining are two suggestions for prevention (Lassus, Tulikoura, Konttinen, Salo, \& Santavirta, 2002). At the first sign of any possibility of a stress injury, workouts should be shortened or modified, replacing a high-impact activity with a lower-impact activity (Lassus, Tulikoura, Konttinen, Salo, \& Santavirta, 2002). Prevention can also occur in the form of nutrition. Adequate nutrition is important for all athletes, however, enough dietary calcium, protein, and energy must be consumed to ensure adequate BMD 
particularly if the athlete is at risk for an eating disorder (Lassus, Tulikoura, Konttinen, Salo, \& Santavirta, 2002).

Along with prevention, correcting any biomechanical factors or the underlying problem in a stress injury are important (Zeni, Street, Dempsey, \& Staton, 2000). If an athlete has a high arch or is flat footed, orthotics may be utilized to help correct this. If the athlete has weak hips causing the knees to become valgus or varus, a strengthening program may be used to correct this. If the athlete has a low BMD or not enough calcium in their diet, this can be corrected as well with appropriate supplements or adjustment in diet. The goal is to not have any complications in the treatment of a stress injury and prevent one from occurring again.

\section{Return to Activity}

Treatment plans previously outlined may help dictate when an athlete should begin a return to activity plan. The overall goal of a return-to-activity plan is to enable an athlete to return to the level of activity he or she was engaged in prior to the stress injury.

Once the athlete is allowed to begin returning to activity, pain is the main feedback in the process. It is important to educate the athlete in the fact that this return process is a graded, stepwise journey, and that it may take as long as the immobilization or period of active rest did. One return to activity model in the literature is the three phase treatment and management plan based on the cyclic process of bone remodeling (two harder weeks followed by one rest week) (Romani, Gieck, Perrin, Saliba, \& Kahler, 2002). Phase III is dependent on being pain-free for two weeks in the previous phase (Phase II included general conditioning and specific strengthening of the injured region, 
while Phase I involved treatment modalities to manage pain and ROM exercises), and includes functional and running activities (Romani, Gieck, Perrin, Saliba, \& Kahler, 2002). Suggestions are to increase activity no more than $15 \%$ to $20 \%$ per week and to participate in a "walk-jog" (Romani, Gieck, Perrin, Saliba, \& Kahler, 2002). A walk-jog is when the athlete returning to activity jogs the straights of a track and walk the curves for 0.5 miles, followed by a day of rest, which is increased in distance when the athlete is pain free to the activity, and then walk-jogs can be performed three times per week (Romani, Gieck, Perrin, Saliba, \& Kahler, 2002). This sounds like a sport specific activity that would be effective for track and field athletes. Based off of the three-phase protocol, the third week will be a rest phase, due to newly forming osteocytes and maturing periosteum (Romani, Gieck, Perrin, Saliba, \& Kahler, 2002). Properly educating the athlete about attending to feedback he or she receives during this process will improve the probability of success.

Once the athlete is fully released by the physician to participate in activities, it is important to monitor the training regimen and ensure that no sudden or abrupt changes are made. If the athlete experiences symptoms again at any time, a follow-up visit with a physician should be arranged.

\section{Further Research}

There are clear gaps in the research related to stress injuries. More information is needed about navicular stress reactions after standardized non weight-bearing cast standardized treatment (Burne, Mahoney, Forster, Koehle, Taunton, \& Khan, 2005). One systematic review concluded that the quality and number of studies involving the 
prevention and treatment of lower extremity stress fractures is lacking (Shaffer \& Uhl, 2006). Another review also indicated that there is insufficient evidence about preventative interventions, such as shock-absorbing insole and stretching (Rome, Handoll, \& Ashford, 2009). Research on the epidemiology and etiology of stress injuries is also lacking (Kelsey et al., 2007).

Based on this review of literature, there are many other areas where research is lacking. For example, information about risk factors related to stress injuries is lacking. Numerous risk factors have been suggested, but sufficient evidence to support them has not been provided. This is disappointing, because a clinical history exam conducted by a physician can often reveal information pointing toward the development of a stress injury (e.g., history of stress injury or anorexia nervosa). Additional information about risk factors would allow athletic trainers and other healthcare professionals to be better informed, so that more emphasis could be placed on prevention.

Treatment is another area that needs more support in the literature. There are many suggestions for modalities that may be beneficial, but little to no evidence-based support for their use. It would be helpful to all health care professionals if additional research data were provided about potentially useful treatment modalities.

\section{Conclusion}

There is a wide range of information about overuse injuries important to health care professionals. Understanding the anatomy, risk factors, imaging, treatment and return to activity surrounding the overuse injury of a stress fracture or stress reaction can ensure earlier recognition and treatment of these injuries and possibly prevention. 


\section{References for Case Studies}

Bennell, K., Malcolm, S., Brukner, P., Green, R., Hopper, J., Wark, J., et al. (1998). A 12-month prospective study of the relationshop between stress fractures and bone turnover in athletes. Calcification Tissue International, 63, 80-85.

Bennell, K., Malcolm, S., Wark, J., \& Brukner, P. (1996). Models for the pathogenesis of stress fractures in athletes. British Journal of Sports Medicine, 30, 200-204.

Bradley, W.G. (n.d.) Fundamentals of MRI Part II. Retrieved May 7, 2012, from MRI and CT: http://www.e-radiography.net/mrict/fund\%20mr2/fundmri\%202.htm

Burne, S. G., Mahoney, C. M., Forster, B. B., Koehle, M. S., Taunton, J. E., \& Khan, K. M. (2005). Tarsal Navicular Stress Injury. The American Journal of Sports Medicine, 33, $1875-1881$.

Fredericson, M., Bergman, A. G., Hoffman, K. L., \& Dillingham, M. S. (1995). Tibial stress reaction in runners. The American Journal of Sports Medicine, 23, 472-481.

Giladi, M., Milgrom, C., Simkin, A., \& Danon, Y. (1991). Stress fractures. The American Journal of Sports Medicine, 19, 647-652.

Jones, B. H., Harris, J. M., Vinh, T. N., \& Rubin, C. (1989). Exercise-induced stress fractures and stress reactions of bone: epidemiology, etiology, and classification.

Exercise Sport Science Review, 17, 379-422.

Kelsey, J. L., Bachrach, L. K., Procter-Gray, E., Nieves, J., Greendale, G. A., Sowers, M., et al. (2007). Risk factors for stress fracture among young female cross-country runners. Clinical Investigations , 1457-1463.

Khan, K. M., Brukner, P. D., Kearney, C., Fuller, P.J., Bradshaw, C.J., \& Kiss, Z. S. (1994). Tarsal navicular stress fracture in athletes. Sports Medicine, 17, 65-76.

Kiuru, M., Pihlajamaki, H., \& Ahovuo, J. (2004). Bone stress injuries. Acta Radiologica, $45,317-326$.

Korpelainen, R., Orava, S., Karpakka, J., Siira, P., \& Hulkko, A. (2001). Risk factors for recurrent stress fractures in athletes. The American Journal of Sports Medicine, 29, 304310.

Lassus, J., Tulikoura, I. K., Salo, J., \& Santavirta, S. (2002). Bone stress injuries of the lower extremity . Acta Orthopaedica Scandinavica, 73, 359-368. 
Magnetic Resonance Imaging (MRI). (2009, June 12). Retrieved April 20, 2012, from WebMD mobile: http://www.m.webmd.com/a-to-z-guides/magnetic-resonance-imagingmri

McKenzie, D., Clement, D., \& Taunton, J. (1985). Running shoes, orthotics, and injuries. Sports Medicine, 2, 334-347.

Romani, W. A., Gieck, J. H., Perrin, D. H., Saliba, E. N., \& Kahler, D. M. (2002). Mechanisms and management of stress fractures in physically active persons. Journal of Athletic Training, 37, 306-314.

Rome, K., Handoll, H. H., \& Ashford, R. L. (2009). Interventions for preventing and treating stress fractures and stress reactions of bone of the lower limbs in young adults. The Cochrane Collaboration, 1, 1-42.

Shaffer, S. W., \& Uhl, T. L. (2006). Preventing and treating lower extremity stress reactions and fractures in adults. Journal of Athletic Training, 41, 466-469.

Staff, M. C. (2012, January 26). Bone Scan. Retrieved April 20, 2012, from Mayo Clinic: http://www.mayoclinic.com/health/bone-scan/MY00306

Staff, M. C. (2012, March 23). CT scan. Retrieved April 20, 2012, from Mayo Clinic: http://www.mayoclinic.com/health/ct-scan/MY00309

Sterling, J. C., Edelstein, D. W., Calvo, R. D., \& Webb II, R. (1992). Stress fractures in the athlete. Sports Medicine, 14, 336-346.

X-ray. (2012, March 21). Retrieved April 20, 2012, from MedlinePlus:

http://www.nlm.nih.gov/medlineplus/ency/article/003337.htm

Zeni, A. I., Street, C. C., Dempsey, R. L., \& Staton, M. (2000). Stress injury to the bone among women athletes. Tough Topics in Sports Medicine, 11, 929-947.

\section{References for Literature Review}

Bennell, K., Malcolm, S., Wark, J., \& Brukner, P. (1996). Models for the pathogenesis of stress fractures in athletes. British Journal of Sports Medicine, 30, 200-204.

Burne, S. G., Mahoney, C. M., Forster, B. B., Koehle, M. S., Taunton, J. E., \& Khan, K. M. (2005). Tarsal navicular stress injury. The American Journal of Sports Medicine, 33, $1875-1881$. 
Fredericson, M., Bergman, A. G., Hoffman, K. L., \& Dillingham, M. S. (1995). Tibial stress reaction in runners. The American Journal of Sports Medicine, 23, 472-481.

Frost, H. M. (1994). Wolff's Law and bone's structural adaptations to mechanical usage: an overview for clinicians. The Angle Orthodontist, 64, 175-188.

Jones, B. H., Harris, J. M., Vinh, T. N., \& Rubin, C. (1989). Exercise-induced stress fractures and stress reactions of bone: epidemiology, etiology, and classification. Exercise Sport Science Review, 17, 379-422.

Kelsey, J. L., Bachrach, L. K., Procter-Gray, E., Neives, J., Greendale, G. A., Sowers, M., et al. (2007). Risk factors for stress fracture among young female cross-country runners. Medicine and Science in Sports and Exercise , 1457-1463.

Khan, K. M., Brukner, P. D., Kearney, C., Fuller, P. J., Bradshaw, C. J., \& Kiss, Z. S. (1994). Tarsal navicular stress fracture in athletes. Sports Medicine, 17, 65-76.

Kiuru, M., Pihlajamaki, H., \& Ahovuo, J. (2004). Bone stress injuries. Acta Radiologica, 45, 317-326.

Korpelainen, R., Orava, S., Karpakka, J., Siira, P., \& Hulkko, A. (2001). Risk factors for recurrent stress fractures in athletes. The American Journal of Sports Medicine, 29, 304310.

Lassus, J., Tulikoura, I., Konttinen, Y. T., Salo, J., \& Santavirta, S. (2002). Bone stress injuries of the lower extremity. Acta Orthopaedica Scandinavica, 73, 359-368.

Marieb, E. N., \& Hoehn, K. (2007). Human Anatomy and Physiology. San Francisco: Pearson Benjamin Cummings.

Romani, W. A., Gieck, J. H., Perrin, D. H., Saliba, E. N., \& Kahler, D. M. (2002). Mechanisms and management of stress fractures in physically active persons. Journal of Athletic Training, 37, 306-314.

Rome, K., Handoll, H. H., \& Ashford, R. L. (2009). Interventions for preventing and treating stress fractures and stress reactions of bone of the lower limbs in young adults. The Cochrane Collaboration, 1, 1-42.

Shaffer, S. W., \& Uhl, T. L. (2006). Preventing and treating lower extremity stress reactions and fractures in adults. Journal of Athletic Training, 41, 466-469.

Sterling, J. C., Edelstein, D. W., Calvo, R. D., \& Webb II, R. (1992). Stress fractures in the athlete. Sports Medicine, 14, 336-346. 
Zeni, A. I., Street, C. C., Dempsey, R. L., \& Staton, M. (2000). Stress injury to the bone among women athletes. Tough Topics in Sports Medicine, 11 , 929-946. 